\title{
Antipruritic Effect of Topical Acetaminophen Gel in Histaminergic and Non-histaminergic Itch Provocation: A Double-blind, Vehicle- controlled Pilot Study
}

Leigh A. NATTKEMPER ${ }^{1}$, Kaining ZHI ${ }^{2}$, Kaeli E. ROMERO ${ }^{1}$, Serena M. SHAH ${ }^{1}$, Teresa JU ${ }^{1}$, Kayla FOURZALI ${ }^{1}$, Rachel Shireen GOLPANIAN ${ }^{1}$, Flor MACQUHAE ${ }^{1}$, Yiong Huak CHAN ${ }^{3}$, David B. LEBO ${ }^{2}$ and Gil YOSIPOVITCH ${ }^{1}$

${ }^{1}$ Dr Phillip Frost Department of Dermatology and Cutaneous Surgery, Miami Itch Center, University of Miami Miller School of Medicine, Miami, FL, ${ }^{2}$ Department of Pharmaceutical Sciences, Temple University School of Pharmacy, Philadelphia, PA, USA and ${ }^{3}$ Biostatistics Unit, Yong Loo Lin School of Medicine, National University of Singapore, Singapore

\begin{abstract}
There is a need for new topical antipruritics that are effective on many types of itch. This study examined the antipruritic efficacy of a new formulation of topical acetaminophen. In vitro skin permeability studies showed that $\mathbf{2 . 5 \%}$ and $\mathbf{5 \%}$ formulations are able to rapidly deliver an adequate amount of the drug into the skin. In a double-blind, vehicle-controlled, randomized study in 17 healthy volunteers, $1 \%, 2.5 \%$ and $5 \%$ acetaminophen gels and a vehicle gel were applied to the skin prior to histaminergic and non-histaminergic itch induction and assessment of thermal pain thresholds. The $\mathbf{2 . 5} \%$ and $\mathbf{5} \%$ gel formulations significantly reduced the itch intensity time course and the area under the curve for both histamine and cowhage itch. No effect was noted on heat pain thresholds and no adverse effects were observed. These results suggest that topical acetaminophen would be a safe and effective overthe-counter medication for itch.
\end{abstract}

Key words: pruritus; antipruritic; acetaminophen; itch model; topical therapy.

Accepted Aug 25, 2021, Epub ahead of print Aug 26, 2021

Acta Derm Venereol 2022; 102: adv00640.

Corr: Leigh A. Nattkemper, Dr Phillip Frost Department of Dermatology and Cutaneous Surgery, Miami Itch Center, University of Miami Miller School of Medicine, 33136 Miami, FL, USA. E-mail: Ixn202@med.miami. edu

I tch or pruritus is a one of the most common skin complaints among dermatological patients and is associated with a multitude of skin and systemic conditions. There have been many developments in oral and systemic therapies for itch (1). However, few topical therapies, which are still the first-line treatment for mild, moderate and localized itch, have been developed in recent years. Currently, the main therapies are topical corticosteroids and antihistamines. Topical corticosteroids have an effect on skin inflammation, but no direct antipruritic mechanism. Topical corticosteroids are also associated with adverse effects of skin atrophy with long-term use. Topical antihistamines have an effect only on acute itch, but have limited or no effect on chronic itch. Less commonly used topical treatments are immunomodulators, such as calcineurin inhibitors and, recently, phosphodiesterase-4 (PDE4) inhibitors, which are extremely

\section{SIGNIFICANCE}

Topical acetaminophen gel was effective against both acute (histamine) and chronic (cowhage) models of induced itch, and did not cause any skin irritation or discoloration. Topical acetaminophen formulas may therefore be a safe and effective over-the-counter therapy for many types of itch.

expensive. Topical anaesthetics, cooling agents, and counter-irritants are used, but these have limited effects on itch. Therefore, there is a great unmet need to develop topical antipruritics that are effective for a large range of pruritic diseases, which are also inexpensive and safe.

Oral acetaminophen is one of the most commonly utilized medications worldwide for pain relief. Although acetaminophen is thought to induce analgesia and antipyresis through its inhibition of the cyclooxygenase pathway and prostaglandin synthesis, its exact mechanism of action has not been fully elucidated. Interestingly, there are no topical acetaminophen formulations on the market, even though a topical formulation could display an antipruritic effect by blocking prostaglandins that are known to potentiate itch (2).

Given this information, the aim of this study was to examine the efficacy of topical acetaminophen formulations on a model of acute and chronic itch in healthy volunteers in a double-blinded, vehicle-controlled, randomized study. A further aim was to determine whether topical acetaminophen formulations have adequate skin permeability and accumulation in the epidermis.

\section{MATERIALS AND METHODS}

Drug formulation

Topical acetaminophen (APAP) was generated into an ointment or gel formulation that compromised $1 \%, 2.5 \%$, and $5 \%$ dose compositions. Vehicle controls were prepared using the same materials and methods without the addition of the active ingredient (APAP).

\section{In vitro skin permeation}

Skin permeation of the formulations $(2.5 \%$ and $5 \%$ APAP; gel and ointment) were tested on cadaver skin, using non-occluded and occluded PermeGear Franz diffusion apparatus (PermeGear, Inc., Hellertown, PA, USA). A $20 \mu 1$ volume of the formulations were added to the donor compartment to form a thin film on the 
surface of the dermatomed human cadaver skin (Anatomy Gifts Registry, Hanover, MD USA). At 1, 2, 4, 8, 12, and 24 h, the drug content in the samples was analysed using the High-performance liquid chromatography (HPLC) method. The steady state flux (J) for permeation $\left(\mu \mathrm{g} / \mathrm{cm}^{2} / \mathrm{h}\right)$ was obtained from the slope of the linear potion of the plot of cumulative amount of APAP permeating per unit surface area of skin $\left(\mu \mathrm{g} / \mathrm{cm}^{2}\right)$ against time $(\mathrm{h})$. The $1 \%$ APAP formulations were not tested for skin permeability, but due to the adequate absorption of the $2.5 \%$ dose, the $1 \%$ formulation was included in the antipruritic efficacy study.

In order to also examine cutaneous retention in the epidermis and dermis, finite dose skin penetration studies of the $5 \%$ formulations were also performed by analysing for drug content by separating the epidermis and the dermis using a modified heat separation method (3). The drug content was extracted from each layer and the concentration of the drug in the extracting medium was quantified using HPLC at the 12-h time-point. The drug levels are reported in $\mu \mathrm{g} / \mathrm{ml}$ to determine the extent of absorption in the epidermis and the dermis.

\section{Subjects}

A total of 17 healthy volunteers were included in the study (age $26.4 \pm 8.1$ years; age range $19-50$ years). Subject demographics are shown in Table I. The participants had no history of pruritic or dermatological diseases and were not using any systemic or topical anti-itch or pain relief agents. All procedures were approved by the University of Miami Institutional Review Board, and written informed consent was provided by each participant. All the 17 screened subjects were enrolled and completed the study.

\section{Design overview}

The study was conducted under double-blinded conditions, with each treatment assigned a code A-D. Subjects participated in 2 study visits within a 1-week time-period, with the only difference between visits being the itch induction technique, which was randomized between cowhage or histamine provocation. At each visit, $4 \times 4 \mathrm{~cm}$ square test areas were demarcated on the volar forearms, 2 on each arm, each separated by $4 \mathrm{~cm}$. Each area was pretreated for 30 min with $0.4 \mathrm{ml}$ volume of 1 of 4 topical gel formulations spread evenly over the test area: APAP $5 \%, 2.5 \%, 1 \%$ or vehicle control gel. The location of each formulation was randomized based on their code, but each subject has the same formulation placement for both visits. This study design allows for each of the 3 study treatments and vehicle to be applied to every subject twice in the same location within 1 week. After 30 min of pre-treatment any remaining gel was wiped away and itch was induced at each of the 4 pre-treated areas consecutively, with at least 5 min between

\section{Table I. Subject demographics}

\begin{tabular}{lr}
\hline Demographics & $n(\%)$ \\
\hline Ethnicity & \\
Hispanic & $5(29)$ \\
Non-Hispanic & $12(71)$ \\
Race & \\
White & $11(64)$ \\
Black or African American & $3(18)$ \\
Asian & $3(18)$ \\
American Indian or Alaska Native & $0(0)$ \\
Native Hawaiian or Other Pacific Islander & $0(0)$ \\
Sex & \\
Female & $15(88)$ \\
Male & $2(12)$ \\
Age & \\
18-35 years & $14(82)$ \\
$36-65$ years & $3(18)$ \\
$>65$ years & $0(0)$ \\
\hline
\end{tabular}

initiation of each treatment. Itch intensity was assessed via numerical rating scale (NRS) every $30 \mathrm{~s}$ following the initiation of itch sensation and recorded until all itch sensation had subsided. Itch duration was also recorded. Heat pain threshold (HPT) assessment, using quantitative sensory testing, was performed on each test area (after itch had subsided for $5 \mathrm{~min}$ ) to examine whether the APAP formulations had any analgesic effect.

\section{Cowhage (non-histaminergic) itch induction}

To induce an acute model of chronic/non-histaminergic itch, approximately 40-45 cowhage spicules were applied and rubbed gently within the test area for $45 \mathrm{~s}(4,5)$. The subjects were instructed to start rating itch intensity on the Itch NRS once they perceived itch and for every $30 \mathrm{~s}$ until the itch subside. Cowhage spicules were then removed using adhesive tape. The NRS was a $0-10$ scale with 0 weighted as "no itch" and 10 weighted as "worst itch imaginable".

\section{Histamine itch induction}

To induce acute histaminergic itch, iontophoresis of a $1 \%$ histamine solution $(4,5) .0 .2 \mathrm{ml}$ of the $1 \%$ histamine solution was placed in an anode delivery electrode placed on the test area, while a cathode grounding electrode was placed on the subject's arm at a certain distance $(<10 \mathrm{~cm}$ away) from the test area (but not in the test area). The iontophoresis device, a battery-powered stimulus isolation unit (Perimed PF 3826, Perilont Power device, Järfälla, Sweden) generates a current of no more than $500 \mu \mathrm{A}$ for $60 \mathrm{~s}$. The NRS was a $0-10$ scale, with 0 weighted as "no itch" and 10 weighted as "worst itch imaginable".

\section{Heat pain thresholds}

$\mathrm{HPT}$, as a measure of unmyelinated $\mathrm{C}$ nerve fibre function, were assessed using the TSA-II neurosensory analyser (Medoc, Ramat Yishay, Israel) at all test sites once itch had subsided for at least 5 min. A thermode probe warmed the skin surface from a baseline of $32^{\circ} \mathrm{C}$ to a maximum of $50^{\circ} \mathrm{C}$ in $0.4^{\circ} \mathrm{C}$ increments. The threshold was determined as a mean of 3 times by the ascending method of limits, where the subject was instructed to press a response button upon detection of a painful stimulus.

\section{Safety measurements}

Erythema was measured by using a Likert scale after pretreatment with the formulations/before itch was induced, $90 \mathrm{~s}$ after itch was perceived, and once the itch subsided, in order to determine whether the APAP formulations caused any skin irritation and whether it reduced any flare caused by the itch inductions. The Likert scale was weighted as follows: $0=$ none, $1=$ barely perceptible, $2=$ slight redness, $3=$ moderate redness, $4=$ heavy or substantial redness, $5=$ extreme redness, and $6=$ severe redness. Any adverse events were also recorded up to 1 week after each subjects' last study visit.

\section{Statistical analysis}

Data were analysed using IBM SPSS. Basic descriptive statistics are reported as mean (standard deviation; SD), range, and $n(\%)$. Statistical significance was set at $p<0.05$. Normality assessment was tested by visual inspection and Shapiro-Wilks tests. Analysis of variance (ANOVA) and repeated measures ANOVAs (when necessary) were used for the main analysis, with post hoc comparisons using the Bonferroni or Fisher's least significant difference correction. Wilcoxon signed-ranks tests was used to analyse erythema scores. 
a

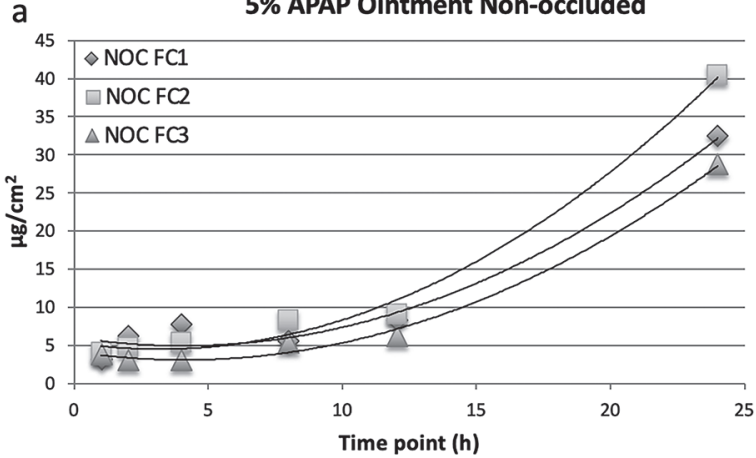

$\mathbf{b}_{80}$

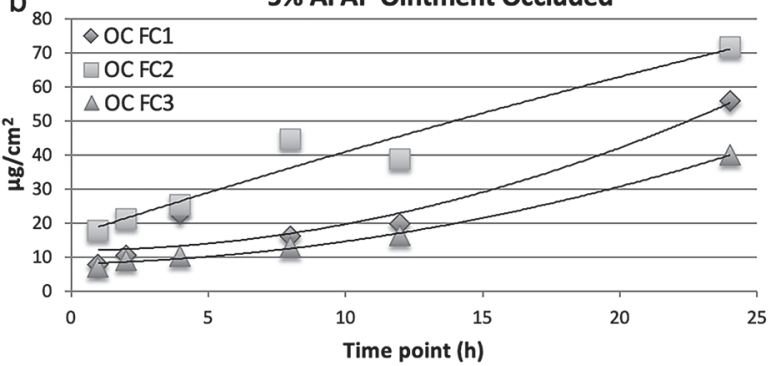

c
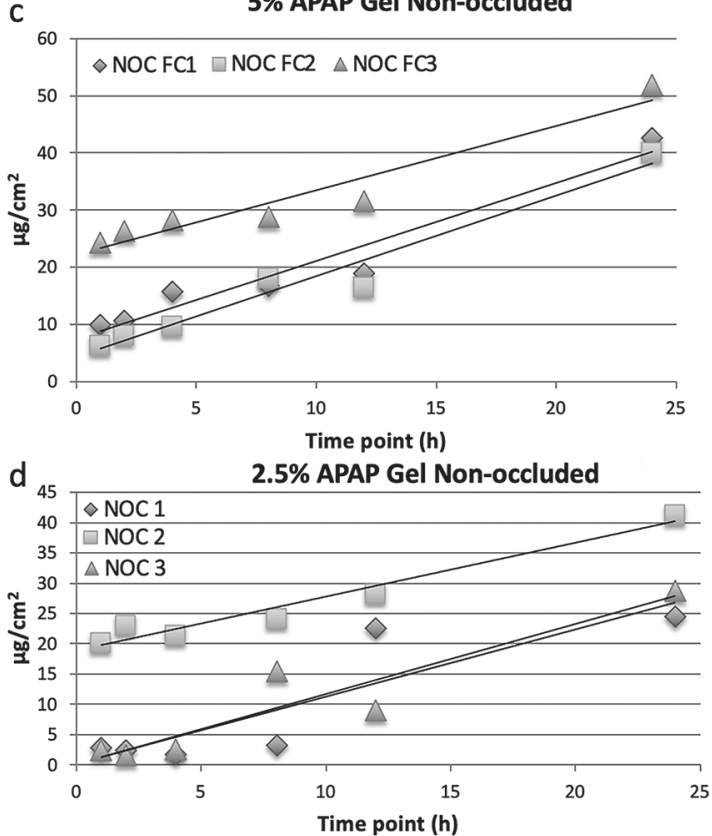

Fig. 1. Skin permeation using Franz cells (FC). (a) The $5 \%$ acetaminophen (APAP) ointment formulation under non-occluded (NOC) conditions provided low skin permeation. (b) The 5\% APAP ointment formulation under occluded (OC) conditions provided approximately $2-20 \mu \mathrm{g} / \mathrm{cm} 2$ in the first hour. (c) The $5 \%$ APAP gel formulation under non-occluded conditions provided the highest permeation at $10-40 \mu \mathrm{g} / \mathrm{cm}^{2}$ in the first hour. (d) The $2.5 \%$ APAP gel formulation under non-occluded conditions provided $2-20 \mu \mathrm{g} / \mathrm{cm}^{2}$ in the first hour, similar to the occluded $5 \%$ APAP ointment. Each symbol represents an individual Franz cell where drug concentration is measured via high-performance liquid chromatography at $1,2,4,8,12$, and 24 h.

\section{RESULTS}

\section{In vitro skin permeation}

The 5\% APAP ointment had low permeation under nonoccluded conditions (Fig. 1a). Low permeation could be due to a partitioning effect, where the APAP may not partition out of the dosage form into the skin. However, under occlusion, the 5\% APAP ointment provided 2-20 $\mu \mathrm{g} / \mathrm{cm}^{2}$ APAP in the first hour (Fig. 1b). The 5\% APAP gel in non-occluded conditions provided approximately $10-40 \mu \mathrm{g} / \mathrm{cm}^{2}$ of APAP within the first hour, while the 2.5\% APAP gel in the same condition and time frame provided 2-20 $\mu \mathrm{g} / \mathrm{cm}^{2}$ (Fig. 1c, d). Occlusion of the Franz apparatus did not improve the initial permeation of gel APAP. The occluded 5\% APAP ointment and nonoccluded $2.5 \%$ APAP gel offered comparable permeation.

Finite dose skin penetration studies revealed that both the non-occluded 5\% ge 1 and the occluded 5\% ointment had greatest concentration of APAP within the epidermis after 12-h treatment (Table II).

Table II. Dose distribution of acetaminophen (APAP) in the skin after 12-h treatment

\begin{tabular}{lcl}
\hline Formulation & Amount $(\mu \mathrm{g} \pm \mathrm{SD})$ & $\%$ (of dose $\pm \mathrm{SD})$ \\
\hline Gel (non-occluded) & $121.9 \pm 30.0$ & \\
Epidermis & $85.7 \pm 9.3$ & $21.5 \pm 9.17$ \\
Dermis & $106.1 \pm 48.3$ & \\
Ointment (occluded) & $69.2 \pm 29.7$ & $35.1 \pm 15.1$ \\
Epidermis & $22.9 \pm 9.2$ \\
\hline
\end{tabular}

SD: standard deviation.

\section{Itch intensity}

Both cowhage and histamine itch intensity, as measured by the NRS, was significantly reduced by the $2.5 \%$ and $5 \%$ gel formulations, as shown in Fig. 2, which depicts the entire time courses of each itch model for all treatment conditions. Itch intensity over time when quantified in the integral measure of area under the curve (AUC), represents the total itch perceived for the full duration. Pretreatment with the 2.5\% and 5\% APAP gel formulations significantly reduced the AUC for both cowhageand histamine-induced itch compared with the vehicle formulation (Fig. 3). The 1\% gel formulation did not significantly reduce the itch intensity time course or the AUC of either cowhage or histamine itch. The peak itch intensity (measured as the max NRS value) was only significantly reduced from the vehicle by the $2.5 \%$ APAP gel formulation during cowhage itch induction $(p=0.003)$, which corresponds to a $32.8 \%$ reduction $(3.9 \pm 2.8$ for $2.5 \%$ APAP gel vs $5.8 \pm 2.6$ vehicle gel). The $5 \%$ APAP gel formulation trended $(p=0.075)$ to reduce the peak itch with a $22.4 \%$ reduction compared with the vehicle during cowhage itch induction $(4.5 \pm 2.0$ for $5 \%$ APAP gel vs $5.8 \pm 2.6$ vehicle gel). Otherwise, peak itch intensity and the total duration of each induced itch types were not significantly affected by the APAP formulations (Fig. 4).

\section{Heat pain thresholds}

Heat pain thresholds were not affected by the APAP gel formulations after either cowhage- or histamine-induced 

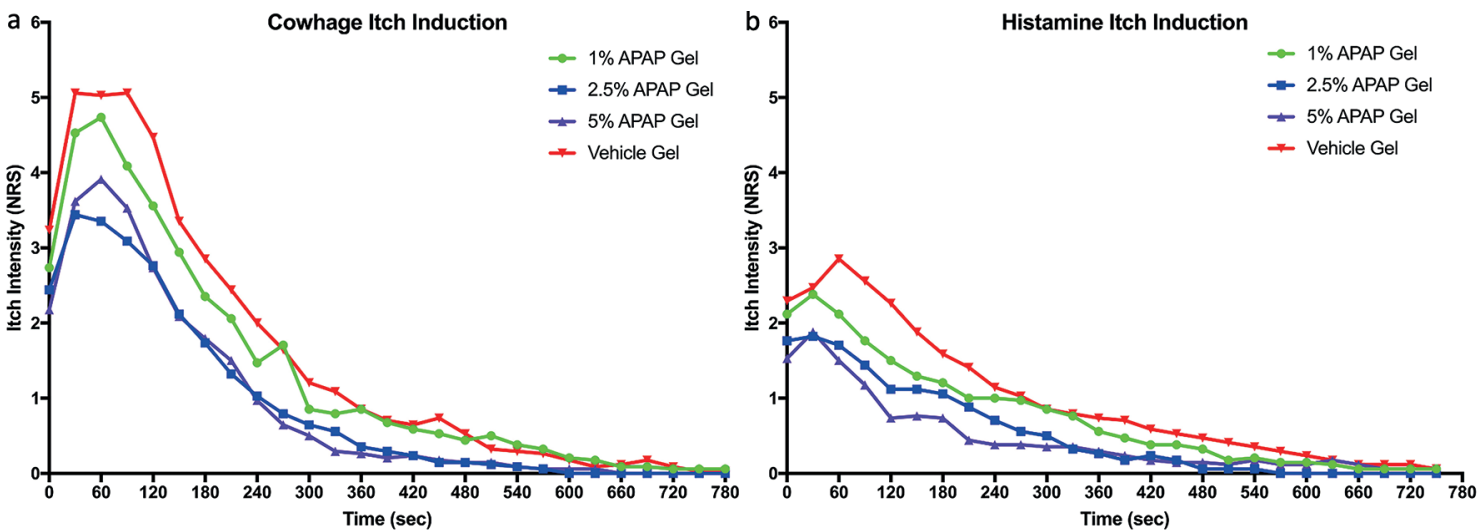

Fig. 2. Itch intensity time course. (a) Each acetaminophen (APAP) gel formulation (1\% $p=0.0081 ; 2.5 \% p<0.0001 ; 5 \% p<0.0001)$ reduced itch intensity compared with the vehicle over the cowhage itch induction time course (repeated-measures analysis of variance (RM-ANOVA) with Bonferroni correction). (b) Each APAP gel formulation $(1 \% p=0.0002 ; 2.5 \% p<0.0001 ; 5 \% p<0.0001)$ reduced itch intensity compared with the vehicle over the histamine itch induction time course.

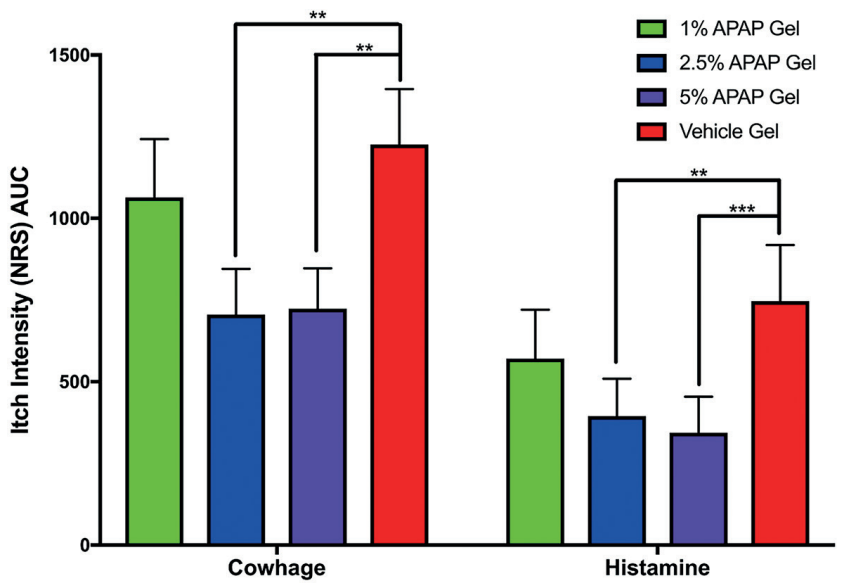

Fig. 3. Itch intensity area under the curve. Total perceived itch, measured as area under the curve (AUC), was significantly reduced by the $2.5 \%$ $\left(p=0.003^{* *}\right)$ and $5 \%\left(p=0.005^{* *}\right)$ acetaminophen (APAP) gel formulation compared with the vehicle in cowhage itch induction. Itch AUC was also significantly reduced by the $2.5 \%\left(p=0.011^{* *}\right)$ and $5 \%\left(p=0.001^{* * *}\right)$ APAP gel formulation compared with the vehicle in histamine itch induction. itch. Furthermore, HPTs were not different between the 2 study visits. This suggests that the formulations do not cause any analgesic or numbing effect (Fig. 5).

\section{Erythema and safety}

Erythema caused by cowhage and histamine itch induction was not affected by the APAP formulations, with no test area being scored over rank 3 of "moderate redness" (Fig. 6). Erythema grades were overall significantly increased $(p=0.001)$ after histamine itch compared with cowhage itch, as is expected due to histamine's associated wheel and flare reaction. Furthermore, application of the formulations (prior to any itch induction) did not cause any erythema (all 0 "none" scores), stinging, skin irritation, or hyper- and hypo-pigmentation. No adverse events were reported for this study.

\section{DISCUSSION}

The current study demonstrated that the acetaminophen gel formulations significantly reduced both histaminergic
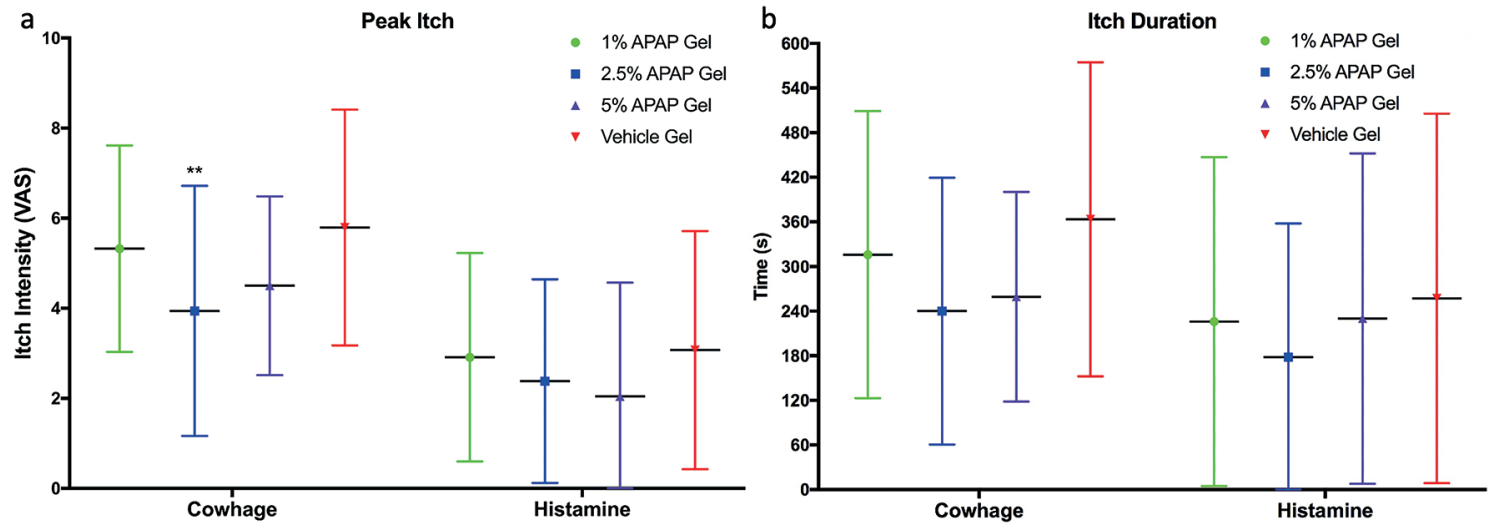

Fig. 4. Itch peak intensity and duration. (a) Peak itch, the maximum itch intensity reported on the numerical rating scale (NRS), was only significantly reduced by the $2.5 \%$ gel acetaminophen (APAP) formulation during the cowhage itch induction compared with the vehicle $\left(p=0.003^{* *}\right)$. Otherwise, no other APAP formulation reduced peak itch. (b) Itch duration, the time the induced itch lasts, was also not affected by any of the APAP formulations with both cowhage and histamine itch induction. 


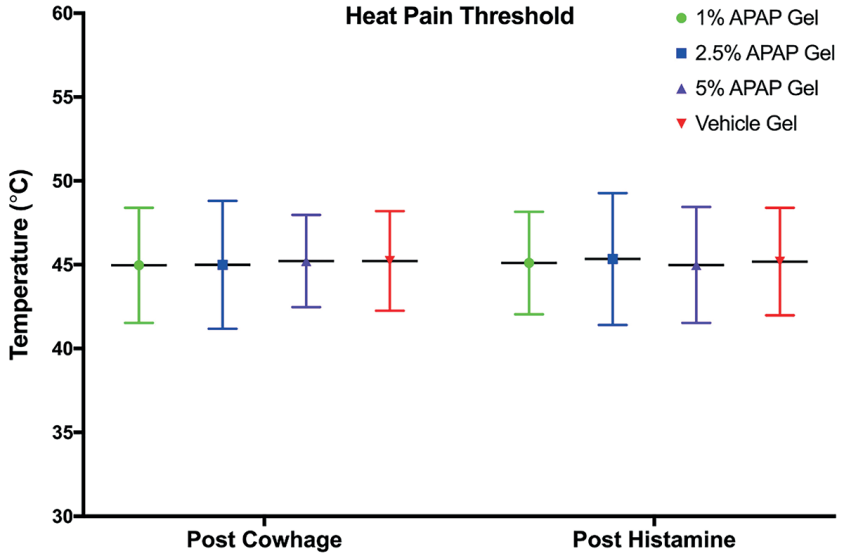

Fig. 5. Heat pain thresholds. Heat pain thresholds, a measure of unmyelinated $\mathrm{C}$ fibre function, were tested by quantitative sensory testing after itch induction, and were not significantly affected by the acetaminophen (APAP) formulations compared with the vehicle for both cowhage and histamine itch induction. Furthermore, heat pain thresholds were not different between itch induction types at different study visits.

and non-histaminergic itch. To our knowledge, there has been no previous study that examined the anti-pruritic effect of acetaminophen in humans, but it has been shown in mice with serotonin-induced itch (6). The induced itch models used in this study mimic real-world acute itch (histaminergic) and chronic itch (non-histaminergic/ cowhage). A study using similar itch models showed that topical polidocanol, an OTC antipruritic marketed in Europe, only reduced cowhage itch, but not histamine itch (7). Also, another study showed that topical aspirin could relieve histamine-induced itch (8). Therefore, it is important to note that this APAP gel formulation is effective for both types of itch. The $2.5 \%$ and $5 \%$ APAP gel formulations were more effective in reducing itch than the $1 \%$ gel formulation. The antipruritic effect noted here is more robust than a previous study we conducted on the effect of topical $4 \%$ strontium on cowhage-induced itch (9).

Studies have suggested that the analgesic relief afforded by acetaminophen involves activation of the cannabinoid system and, indeed, antagonism of the cannabinoid (CB)-1 receptor has been shown to block acetaminophen-induced analgesia $(10,11)$. Given the similarities between pain and itch transmission, it is possible, through its action on $\mathrm{CB} 1$ receptors, that acetaminophen may have antipruritic properties. Expression of the phospholipase-2 (PLA2) family of enzymes involved in the arachidonic acid pathway was found to be upregulated in itchy atopic and psoriatic skin, and was correlated significantly with itch severity (12). As acetaminophen is thought to act along the arachidonic acid pathway, which provides further evidence that this medication may possess an anti-pruritic effect. In addition, metabolites of acetaminophen have been shown to activate and sensitize transient receptor potential (TRP) cation channel subfamily V (TRPV1) receptors, which are known to play an important role in itch transmission and inflammation $(13,14)$. None of the formulations of topical acetaminophen gel exhibited any anti-nociceptive /analgesic effect, which is similar to a previous study of the effect of intravenous acetaminophen on the same type of thermal quantitative sensory testing as described in the current study (15).

Acetaminophen is currently an over-the-counter (OTC) product in the US market. While it is generally well tolerated and is considered a safe medication, adverse effects of oral or intravenously administered acetaminophen include, but are not limited to, gastrointestinal upset, and hepatotoxicity at high doses (16). Subcutaneous administration of acetaminophen for pain relief has been shown to be well tolerated in patients on palliative care (17). The permeation data and lack of any adverse effects caused by application of these acetaminophen gels suggest that this topical formulation would be effective and safe. Furthermore, optimal permeation times suggest that minimal daily treatment applications may be effective, but the safety profile would allow for multiple applications, if required. Future efficacy studies in patients and on long-term application of the acetaminophen
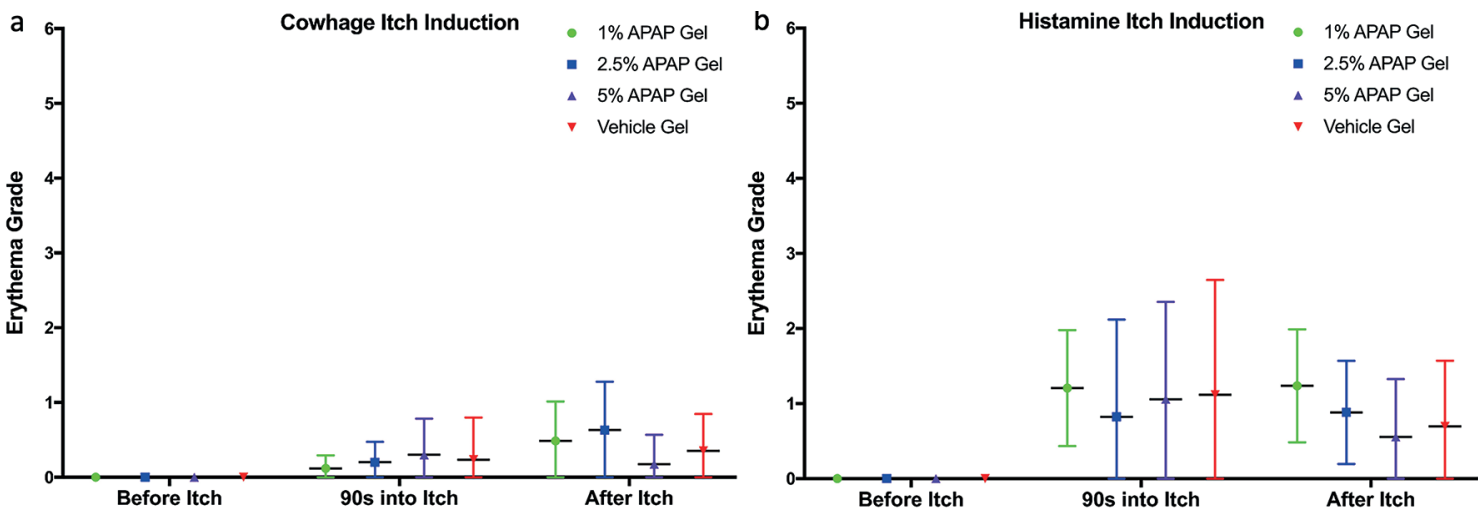

Fig. 6. Erythema ratings. Erythema, as a measure of skin irritation, was ranked on a $0-6$ Likert scale after pretreatment of acetaminophen (APAP) formulations/before itch induction, $90 \mathrm{~s}$ after itch was perception, and after the end of itch perception. The application of the formulations caused no erythema, and the formulations did not significantly affect erythema related to either (a) cowhage or (b) histamine itch induction. Erythema was scored higher with histamine itch induction compared with cowhage at both $90 \mathrm{~s}$ into itch induction and after the itch subsided, due to histamine's expected wheel and flare reaction $(p=0.001)$. 
gels are required to know what types of itch would best respond to this topical treatment. Nevertheless, the $2.5 \%$ or 5\% APAP gel formulation could be offered as another antipruritic option in the OTC market for itch.

\section{ACKNOWLEDGEMIENT}

This study was supported by the University of Miami Wallace H. Coulter Center Translational Research Commercialization Grant to LN, DL and YG.

Conflicts of interest. LAN, DBL and GY have a pending patent for the acetaminophen formulations used in the study.

\section{REFERENCES}

1. Fourzali K, Golpanian RS, Yosipovitch G. Emerging drugs for the treatment of chronic pruritic diseases. Expert Opin Emerg Drugs 2020; 25: 273-284.

2. Greaves MW, McDonald-Gibson W. Itch: role of prostaglandins. Br Med J 1973; 3: 608-609.

3. Kassis V, Søndergaard J. Heat separation of normal human skin for epidermal and dermal prostaglandin analysis. Arch Dermatol Res 1982; 273: 301-306.

4. Papoiu AD, Tey HL, Coghill RC, Wang H, Yosipovitch G. Cowhage-induced itch as an experimental model for pruritus. A comparative study with histamine-induced itch. PLoS One 2011; 6: e17786.

5. Andersen HH, Sørensen A-KR, Nielsen GAR, Mølgaard MS, Stilling $\mathrm{P}$, Boudreau SA, et al. A test-retest reliability study of human experimental models of histaminergic and nonhistaminergic itch. Acta Derm Venereol 2017; 97: 198-207.

6. Saglam G, Gunduz O, Ulugol A. Blockade of cannabinoid CB1 and $C B 2$ receptors does not prevent the antipruritic effect of systemic paracetamol. Acta Neurol Belg 2014; 114: 307-309.

7. Hawro T, Fluhr JW, Mengeaud V, Redoulès $D$, Church MK, Maurer $M$, et al. Polidocanol inhibits cowhage - but not histamine-induced itch in humans. Exp Dermatol 2014; 23: 922-923.

8. Yosipovitch G, Ademola J, Lui P, Amin S, Maibach HI. Topically applied aspirin rapidly decreases histamine-induced itch. Acta Derm Venereol 1997; 77: 46-48.

9. Papoiu AD, Valdes-Rodriguez R, Nattkemper LA, Chan YH, Hahn GS, Yosipovitch G. A novel topical formulation containing strontium chloride significantly reduces the intensity and duration of cowhage-induced itch. Acta Derm Venereol 2013; 93: 520-526.

10. Ottani A, Leone S, Sandrini M, Ferrari A, Bertolini A. The analgesic activity of paracetamol is prevented by the blockade of cannabinoid CB1 receptors. Eur J Pharmacol 2006; 531: 280-281.

11. Klinger-Gratz PP, Ralvenius WT, Neumann E, Kato A, Nyilas $R$, Lele $Z$, et al. Acetaminophen relieves inflammatory pain through CB1 cannabinoid receptors in the rostral ventromedial medulla. J Neurosci 2018; 38: 322-234.

12. Nattkemper LA, Tey HL, Valdes-Rodriguez R, Lee $H$, Mollanazar NK, Albornoz C, et al. The genetics of chronic itch: gene expression in the skin of patients with atopic dermatitis and psoriasis with severe itch. J Invest Dermatol 2018; 138 : 1311-1317.

13. Valdes-Rodriguez R, Kaushik SB, Yosipovitch G. Transient receptor potential channels and dermatological disorders. Curr Top Med Chem 2013; 13: 335-343.

14. Eberhardt MJ, Schillers F, Eberhardt EM, Risser L, de la Roche J, Herzog C, et al. Reactive metabolites of acetaminophen activate and sensitize the capsaicin receptor TRPV1. Sci Reports 2017; 7: 1-18.

15. Tiippana E, Hamunen K, Kontinen V, Kalso E. The effect of paracetamol and tropisetron on pain: experimental studies and a review of published data. Basic Clin Pharmacol Toxicol 2013; 112: 124-131.

16. Ramachandran A, Jaeschke $H$. Acetaminophen hepatotoxicity. Semin Liver Dis 2019; 39: 221-234.

17. Leheup BF, Ducousso S, Picard S, Alluin R, Goetz C. Subcutaneous administration of paracetamol - good local tolerability in palliative care patients: an observational study. Palliat Med 2018; 32: 1216-1221. 